\title{
Adjectives Grouping in a Dimensionality Affective Clustering Model for Fuzzy Perceptual Evaluation
}

\author{
Wenlin Huang ${ }^{1}$, Qun Wu²,34*, Nilanjan Dey ${ }^{5}$, Amira S. Ashour ${ }^{6}$, Simon James Fong 7,8 , Rubén González Crespo 9,10 \\ ${ }^{1}$ School of Media \& Art Design, Wenzhou Business College, Wenzhou (PR China) \\ ${ }^{2}$ Institute of Universal Design, Zhejiang Sci-Tech University, Hanzhou (PR China) \\ ${ }^{3}$ Collaborative Innovation Center of Culture, Creative Design and Manufacturing Industry of China Academy of Art, Hangzhou \\ (PR China) \\ ${ }^{4}$ Zhejiang Provincial Key Laboratory of Integration of Healthy Smart Kitchen System, Hangzhou (PR China) \\ ${ }^{5}$ Department of Information Technology, Techno International New Town, West Bengal (India) \\ ${ }^{6}$ Department of Electronics and Electrical Communications Engineering, Faculty of Engineering, Tanta University (Egypt) \\ ${ }^{7}$ Department of Computer and Information Science, University of Macau, Macau SAR (China) \\ ${ }^{8}$ DACC Laboratory, Zhuhai Institutes of Advanced Technology of the Chinese Academy of Sciences (China) \\ ${ }^{9}$ Department of Computer Science and Technology, Universidad Internacional de La Rioja, Logroño \\ (Spain) \\ ${ }^{10}$ Department of Computer Science and Technology, Marconi International University / UNIR LLC, \\ Miami, Florida (USA) \\ Received 23 March 2020 | Accepted 25 May 2020 | Published 27 May 2020

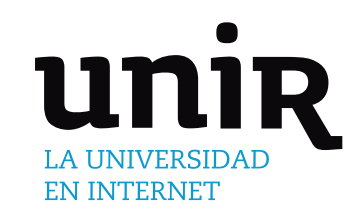

\section{ABSTRACT}

More and more products are no longer limited to the satisfaction of the basic needs, but reflect the emotional interaction between people and environment. The characteristics of user emotions and their evaluation scales are relatively simple. This paper proposes a three-dimensional space model valence-arousal-dominance (VAD) based on the theory of psychological dimensional emotions. It studies the clustering and evaluation of emotional phrases, called VAdC (VAD-dimensional clustering), which is a kind of the affective computing technology. Firstly, a Gaussian Mixture Model (GMM) based information presentation system was introduced, including the type of the presentation, such as single point, plain, and sphere. Subsequently, the border of the presentation was defined. To increase the ability of the proposed algorithm to handle a high dimensional affective space, the distance and inference mechanics were addressed to avoid lacking of local measurement by using fuzzy perceptual evaluation. By comparing the performance of the proposed method with fuzzy c-mean (FCM), k-mean, hard -c-mean (HCM), extra fuzzy c-mean (EFCM), the proposed VADdC performs high effectiveness in fitness, inter-distance, intra-distance, and accuracy. The results were based on the dataset created from a questionnaire on products of the Ming style chairs online evaluation system.

\section{KEYWORDS}

\section{Affective Computing,} Product Evaluation, Fuzzy Set, Clustering, Valence-ArousalDominance.

\section{INTRODUCTION}

$\mathrm{P}$ ERCEPTUAL engineering is a new branch of engineering which combines the perceptual and engineering technologies. It mainly designs products by analyzing human sensibility and manufactures products according to human preference [1]-[2]. The sensibility of perceptual engineering is a dynamic process, which changes fashion, trend and individual timely. It is difficult to grasp and quantify the perceptual issues, but they can be measured, quantified, and analyzed by modern technology, and their rules still can be grasped [3]. Some uncertain inference with evaluations was widely applied in the industrial design field, especially, for the products preference evaluation [4]-[6]. Researchers from Hiroshima University were the first to introduce

\footnotetext{
* Corresponding author.

E-mail address:wuq@zstu.edu.cn
}

perceptual analysis into the field of engineering research. In 1970, with the beginning of the comprehensive consideration of the emotions and desires of occupants in residential design, the study of how to embody the sensibility of occupants into engineering technology in residential design was originally called "emotional engineering" [7].

The customer's emotional evaluation of the product exists in its natural language description, while the vocabulary in natural language is often inaccurate and vague [8]-[11]. The difficulty in dealing with natural language also complicates the study of implicit emotions in products. The traditional fuzzy set theory coarse-grained natural language and the formation of linguistic variables [12] reduce the computational complexity, which brings a feasible direction to language processing under weakening conditions [13]-[15]. Osgood's semantic difference work is composed of three-dimensional indicators [16]-[18]. One important dimension is the range of "Valence" from pleasant to unpleasant; the other dimension is "Arousal", which measures of calm 
to excited; and "dominance" means the perceived degree of control in a (social) situation, so-called VAD model. Mehrabian and Russell used 150 words for experiments, and Bellezza et al. used 450 words for experiments; Bradley \& Lang launched the psychological quantity calibration experiment to launch an emotional rating data set of about 1000 words, and more than 1000 words were divided into 56 groups, each group consisted of 14 lines of 4 words per line for the subject to rate [19]-[20]. The corresponding vocabulary of the 56 vocabularies is subjected to the three-dimensional sentiment rating of VAD, which is set to a discrete scale [21].

By statistically calculating the data, the VAD emotional space mean and its standard deviation (SD) of each vocabulary are calculated separately. Since the words of the emotional vocabulary are not all suitable for product and perceptual evaluation, some of the vocabularies need to be selected to reflect the product. Emotional elements are carried; therefore, perceptual engineering classifies and merges certain words using grouping, and forms about 25 representative words (perceptual vocabulary groups) through group computing of perceptual vocabulary. This work conducted an emotional rating experiment based on 25 vocabularies and modified the Self-Assessment Manikin (SAM) and Affective Norms for English Words (ANEW) accordingly. Because the traditional SAM-based class experiments use pure manual methods, they face the problem of inefficient data collection, which also imposes constraints on the design of SAM sentiment rating experiments [8]. For example, a discrete scale design is mainly to consider statistical convenience. In this experiment, the expression scale of SAM is still used, but when setting the number of rating points, the continuous point method is adopted, which is also in line with Osgood's theory of "continuous psychological quantity".

In this study, a SAM continuous-scale sentiment rating system was proposed. The data points are implemented using scroll bars, so that the acquired VAD sentiment spatial data is no longer restricted to the discrete distribution of [0-8]. This will be beneficial to discover the microscopic mode of the VAD space; where, the point set distribution form in the VAD space. The purpose of our experiment is to find the relationship between the perceptual vocabulary and the corresponding VAD space, and the distribution of each perceptual vocabulary and VAD emotional space point set, and to find the consistency degree of the emotional vocabulary from the analysis of the distribution state of the point set [22]-[25]. Specifically, the feelings of the user are relatively consistent; these are all expected to be obtained in the experimental data and later analysis. Similarly, in the specific application, the feature set image of the product can be used to perform the same VAD rating experiment to obtain the VAD data of a certain product feature, so that the product's characteristics and emotional elements pass the VAD emotion.

Visually it reproduces the VAD sentiment data of 25 vocabularies; thus, the rating data of the single dimension is drawn separately. The results established that each dimension data has an aggregation effect, which means only from a single dimension (Valence, Arousal or Dominance) ( $\mathrm{K}=$ Control), the rating data has certain stability; and from the two-dimensional data point distribution observation ( $\mathrm{K}=$ Elegant), the data still shows a certain aggregation effect. As the number of data increases, people's ratings are not only stable, but also related to Valence, Arousal and Dominance. However, they cannot be generalized by linear regression. The traditional method is to use cluster analysis to select representative point sets for classification, but the results of clustering still make each emotional element become an isolated point [26]-[27].

In this research, the VAD emotional space was constructed by using the modeling proposed in Section II. Two clustering algorithms were applied for emotion clustering and presented the ANEW system words in VAD space, the 3-dimensional emotion space that was potentially applied in an industrial design product evaluation by using a fuzzy inference system. Section III deployed the results and discussions, while Section IV concluded the proposed methods effectively in some applications. The framework of the research is illustrated in Fig. 1.

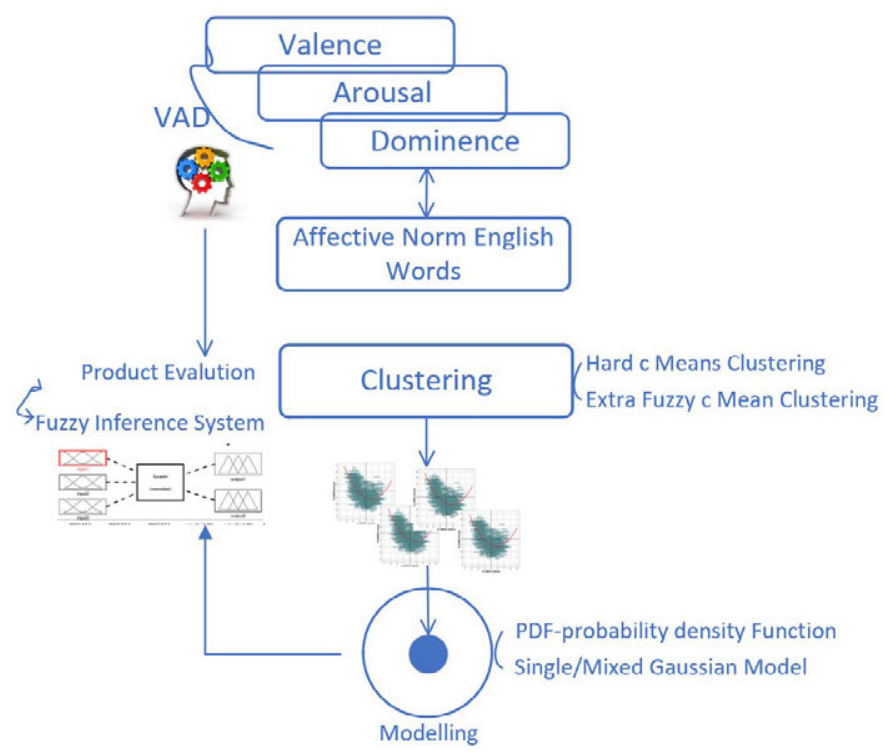

Fig. 1. The framework of VAD emotional model for evaluation by using dimensional clustering methods.

\section{MODELING}

The emotional evaluation by using a perceptual vocabulary is more concentrated and the emotional meta-core can be defined as a single -point set. The center is the average of the three-dimensional coordinates of the point set. The standard deviation is the domain radius. The point set distribution has a certain range, which can be calculated by its standard deviation. This kind of data distribution can set the kernel of the emotional element as an ellipsoid set indicating that the VAD identity of the emotional element is general. The VAD point set data is a distributed approximation on a plane indicating the VAD identity of the emotional element, such as a specific 25 -word VAD point set topology. The content is to carry out the artificial psychological quantity labeling experiment based on the VAD emotional space, and the system design is carried out according to the method of ANEW experimental design. Due to the traditional manual labeling method, it is not conducive to large-scale data acquisition, and there are statistical difficulties. The continuous scale method (VAD emotion rating)does no longer adopt the 9-point rating system through the online survey system. VAD labeling experiments can be performed on a large scale to collect more data. The VAD labeling of the product and the VAD labeling of the relevant sensible vocabulary can establish a kind of mapping of the product feature set to the corresponding sensible vocabulary while it is not a one-to-one correspondence function.

The VAD sentimental spatial point set of each perceptual vocabulary shows that the distribution state of VAD spatial point sets of different perceptual vocabularies is different, which can be roughly divided into three categories: single point set (indicating that the emotional evaluation has a strong one), plane set (indicating that the sentiment evaluation is poorly consistent), and ellipsoid set (indicating that the sentiment evaluation has a medium consistency). Through the different spatial geometric topologies of the point set distribution, these emotions need to be defined separately. The emotional cell metamodel is a very special semantic cell model whose domain is a three-dimensional VAD emotional space. As a special semantic cell, 
it is composed of two parts: the semantic kernel and the semantic shell. The semantic kernel is a set of VAD values, which represent the typical VAD values of all emotional cell elements. The emotional cell element shell represents the boundary of the field covered by the perceptual vocabulary, which is essentially uncertain, so a distance density function is used to represent this uncertainty. For the kernel of emotional cell elements, there are many forms, such as single point set, and sphere set. For the outer shell of the emotional cell elements, there are also many forms of the density function, such as the Gaussian Mixture Model (GMM).

\section{A. Adjectives Space Construction}

$\forall P \in \Omega$ is of $\Omega=\left\{\left(E_{1}, E_{2}, \ldots, E_{n}\right): E_{i} \in P . i=1,2, \ldots, n\right.$ where $n$ is the number evaluations. Particularly, in the valence-arousal-dominent dimentinality space, $P$ is described as $P=($ Valence, Arousal, Dominance $)$ and simplified as $P=(v, a, d)$, where $\Omega=\{(v, a, d) \mid v, a, d \in R\}$. A given metric $d=\|\cdot\|$ in VAD dimentionality can be defined as [26]:

$$
\begin{aligned}
& d(P, P)=\|P\|=\sqrt{\left(v^{2}+a^{2}+d^{2}\right)} \\
& \forall P, Q \in \Omega, \text { we have that, } \\
& d(P \pm Q)=\sqrt{\left(\left(v_{P} \pm v_{Q}\right)^{2}+\left(a_{P} \pm a_{Q}\right)^{2}+\left(d_{P} \pm d_{Q}\right)^{2}\right)}
\end{aligned}
$$

Besides, $\forall \alpha, \beta \in R, P, Q \in \Omega, d$ can be given by:

$$
\begin{aligned}
& d(\alpha P \pm \beta Q) \\
& =\sqrt{\left(\alpha v_{P} \pm \beta v_{Q}\right)^{2}+\left(\alpha a_{P} \pm \beta a_{Q}\right)^{2}+\left(\alpha d_{P} \pm \beta d_{Q}\right)^{2}}
\end{aligned}
$$

Thus, the following expression is applied:

$$
d(\alpha P+\beta Q) \leq|\alpha| \cdot d(P)+|\beta| \cdot \mid d(Q)
$$

Definition 1: $\forall P \in \Omega$, there exists a neighbor of $P$ which is defined as:

$$
N_{P}^{\varepsilon}=\{X \mid\|P-X\|<\varepsilon, X \in \Omega\}
$$

Definition 2: For adjectives $K$, if the VAD values belong to a single point kernel, then the kernel is defined by:

$$
\left\{P_{K} \mid P_{K}=\frac{1}{\|K\|} \sum_{P_{i} \in K} P_{i}\left(\rho\left(P_{i}\right)\right)\right\}
$$

where, $\rho\left(P_{i}\right)$ is the probability density function (PDF) of $P_{i}$.

Definition 3: The sphere kernel is defined as:

$$
\left\{P_{j} \mid P_{j} \in N_{P_{K}}^{\varepsilon}\right\}
$$

where, $P_{K}=\frac{1}{\left\|K^{\prime}\right\|} \sum_{P_{i} \in K^{\prime}} P_{i}(\rho(P i)), K^{\prime} \subset K$,

and $K^{\prime}=\left\{P_{i} \mid \rho\left(P_{j}\right) \leq \rho_{T}\right\}, \rho_{T}$ is a given constant to limit the size of the kernel.

Definition 4: The plain kernel is defined as a union of sphere kernels $\left\{\bigcup_{i} P_{K}\right\}$, where $P_{K}$ is subject to Definition 2 and 3 .

Definition 5: The border of the kernel is defined by upper and lower sets, which are respectively given by:

$$
\begin{aligned}
& U P_{B}=\left\{P_{l} \mid P_{l} \in N_{P_{K}}^{\varepsilon_{u}}\right\} \\
& L P_{B}=\left\{P_{l} \mid P_{l} \in N_{P_{K}}^{\varepsilon_{l}}\right\}
\end{aligned}
$$

Then, the border is given by:

$$
P_{B}=U P_{B} \backslash L P_{B}
$$

\section{B. Metric Function Acquisition}

\section{Linear Based Function}

The density calculation of a point set is a relatively complicated process, and a linear function can be used to simplify the calculation. As the constant function $\rho_{i}(x)=a_{i}$, it is clear that when the density function is linear, it will reflect the uniform distribution state of the point set. Fig. 2 shows the grouping of the adjective, where (a) control, and (b) modern in the perceptual space of emotions in the VAD model.

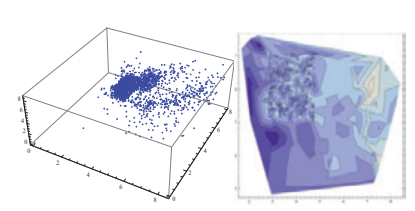

(a)

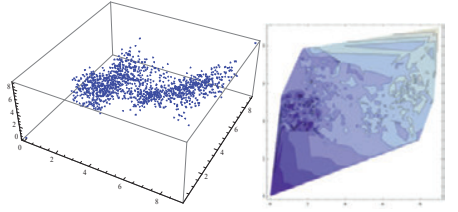

(b)
Fig. 2. The density distribution (middle) and contour map (right) of the linear density function of the VAD spatial point, where (a) $\mathrm{K}=$ control, and (b) $\mathrm{K}=$ modern.

\section{Gaussian Based Metric}

In the point set topology type of the VAD emotion space, both the single point set and the spheroid set are considered to be approximately spherical. In this case, the Simple Gaussian Model (SGM) is used to describe the probability density of these points, which is defined as follows:

$$
\rho(P ; \mu, \Delta)=\frac{1}{\sqrt{(2 \pi)^{3}|\Delta|}} e^{-\frac{1}{2}(P-\mu)^{T} \Delta^{-1}(P-\mu)}
$$

where $\Delta$ is the covariance matrix, and $\mu$ is the center point of the density function. The characteristics of the density function are determined by $(\Delta, \mu)$. Then, to achieve the best description of the point set to feature, the parameter $\mathrm{p}$ of $(\Delta, \mu)$ should be estimated. For any $P_{i} \in \Omega$ of the VAD space, the density probability is $\rho\left(P_{i}, \mu, \Delta\right)$, for any adjective $K$, each point $P_{i} \in K$ is independent, then probability density of $K$ can be calculated by:

$$
\rho_{K}=\rho(K ; \mu, \Delta)=\prod_{i} \rho\left(P_{i} ; \mu, \Delta\right)
$$

By using the maximum likelihood estimation (MLE), the estimating parameter pairs of $(\Delta, \mu)$ to be applied for the maximization are calculated using the following formula, in which $O$ is an estimation on $(\mu, \Delta)[26]$ :

$$
\begin{aligned}
O(\mu, \Delta) & =\ln \left(\prod_{i} \rho\left(P_{i} ; \mu, \Delta\right)\right) \\
& =\sum_{i} \ln \left(\rho\left(P_{i} ; \mu, \Delta\right)\right) \\
& =\sum_{i}\left[-\frac{3}{2} \ln (2 \pi)-\frac{1}{2} \ln |\Delta|+\frac{1}{2}\left(P_{i}-\mu\right)^{T} \Delta^{-1}\left(P_{i}-\mu\right)\right. \\
& \left.=-\frac{3 n}{2} \ln (2 \pi)-\frac{n}{2} \ln |\Delta|-\frac{1}{2} \sum_{i}\left[P_{i}-\mu\right)^{T} \Delta^{-1}\left(P_{i}-\mu\right)\right]
\end{aligned}
$$

To get $\mu$, continuously, equate the differentiation of $\mathrm{Eq}(13)$ by the variable $\mu$ by 0 as follows:

$$
\begin{aligned}
\partial_{\mu}(O(\mu, \Delta)) & =-\frac{1}{2} \sum_{i}\left[-2 \Delta^{-1}\left(P_{i}-\mu\right)\right] \\
& =\Delta^{-1} \sum_{i}\left[\left(P_{i}-\mu\right)\right] \\
& =\Delta^{-1} \sum_{i} P_{i}-n \mu=0
\end{aligned}
$$


Also, the estimated $\mu$ and $\Delta$ are given by:,

$$
\begin{aligned}
& \hat{\mu}=\frac{1}{n} \sum_{i} P_{i} \\
& \hat{\Delta}=\frac{1}{n-1} \sum_{i}\left(P_{i}-\hat{\mu}\right)\left(P_{i}-\hat{\mu}\right)^{T}
\end{aligned}
$$

Any density of the point in the kernel can be presented formally as $\rho(P ; \hat{\mu}, \hat{\Delta})$. The definition of VAD and the center point's parameter estimation $\mu$ can be rewritten as:

$$
\hat{\mu}=\left(\frac{1}{n} \sum_{i} v_{i}, \frac{1}{n} \sum_{i} a_{i}, \frac{1}{n} \sum_{i} d_{i}\right)
$$

The parameter estimation of covariance $\hat{\Delta}$ can be rewritten as:

$$
\begin{aligned}
\hat{\Delta} & =\frac{1}{n-1} \sum_{i}\left[v_{i}-\hat{\mu}_{1}, a_{i}-\hat{\mu}_{2}, d_{i}-\hat{\mu}_{3}\right]\left[\begin{array}{c}
v_{i}-\hat{\mu}_{1} \\
a_{i}-\hat{\mu}_{2} \\
d_{i}-\hat{\mu}_{3}
\end{array}\right] \\
& =\frac{1}{n-1} \sum_{i}\left[\left(v_{i}-\hat{\mu}_{1}\right)^{2}+\left(a_{i}-\hat{\mu}_{2}\right)^{2}+\left(d_{i}-\hat{\mu}_{3}\right)^{2}\right]
\end{aligned}
$$

where $n=2^{P}$, i.e. the number of elements of the set; where the discreet scale can be used if the VAD is in one-dimensional space, where the mode visualization is shown in Fig. 3.

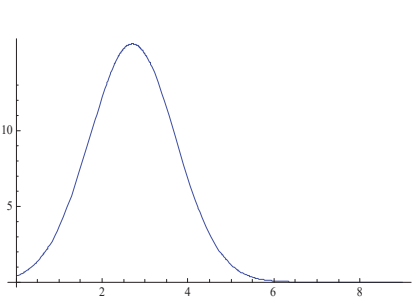

(a)

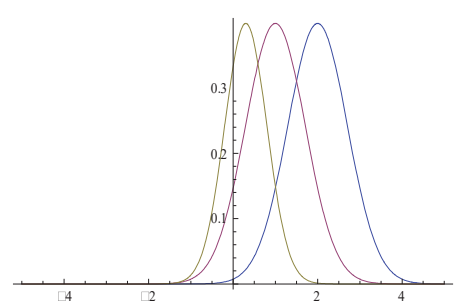

(b)
Fig. 3. One dimensional affective SGM model.

By using $\mathrm{K}=$ Elegant in the VAD dataset, after SGM, we have $\mu=(2.71,4.79,5.75), \Delta=2.07 I$, which is visualized in Fig. 4, where

$$
\begin{aligned}
& \rho\left(P_{\text {Elegant }}\right)=(2 \pi)^{-\frac{3}{2}} e^{-\frac{1}{2}\left((x-2.71)^{2}+(y-4.79)^{2}\right)} \\
& \rho\left(P_{\text {Elegant }}\right)=(2 \pi)^{-\frac{3}{2}} e^{-\frac{1}{2}\left((x-1.70)^{2}+(y-2.45)^{2}\right)}
\end{aligned}
$$

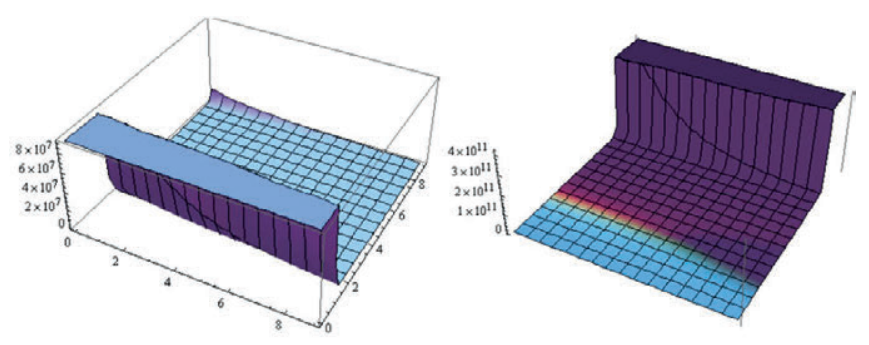

Fig. 4. SGM model in VAD space.

For " $P$ is elegant" in VAD is visualized in Fig. 5, where

$$
\rho\left(P_{\text {Elegant }}\right)=(2 \pi)^{-\frac{3}{2}} e^{-\frac{1}{2}\left((x-2.71)^{2}+(y-4.79)^{2}+(z-5.75)\right)^{2}}
$$

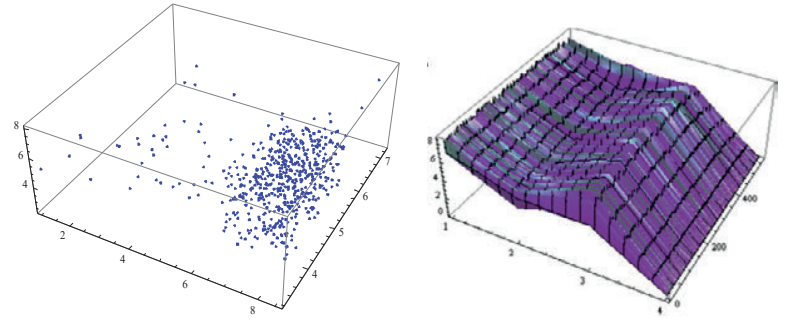

Fig. 5. VAD effective space by using the metric of $\theta=(2.71,4.79,5.75, I)$ and $n=568$.

The kernel of the plane set form is formed by the VAD emotional space, which considered that the recognition of the corresponding emotional vocabulary is low. Thus, the GMM describes the characteristics of the VAD emotional space efficiently. To describe the density value distribution corresponding to the equivalent dimension value, the plot of the Valence Arousal dimension is shown in Fig. 6.

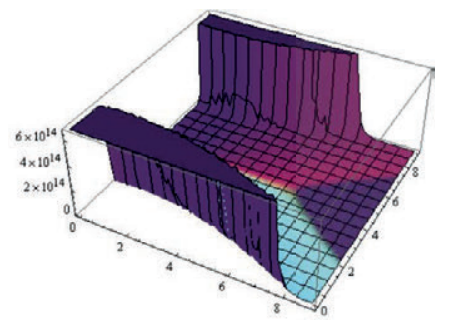

$$
\rho(P)=(2 \pi)^{-\frac{3}{2}}\left(e^{-\frac{1}{2}\left((x-1.71)^{2}+(y-2.79)^{2}\right)}+e^{-\frac{1}{2}\left((x-3.05)^{2}+(y-5.25)^{2}\right)}+e^{-\frac{1}{2}\left((x-1.05)^{2}+(y-7.25)^{2}\right)}\right) .
$$

Fig. 6. The VAD of the three kernels combination in the SGM distribution.

\section{The Density Function of the Single Dimension Affective}

For the VAD emotional space, it is not yet possible to accurately obtain the correlation between the three-dimensional emotional data. However, from the medical observation of the brain magnetic resonance imaging data, there is a certain correlation between VAD. Traditional perceptual engineering uses single-dimensional emotional rating data. When the data point set is on the VAD axis, it means that a certain perceptual semantics presents a one-dimensional emotion. For theoretical completeness, this section gives a method for estimating the density of single-dimensional emotions in a single point set [28]-[32]. The point set of the coordinate axes $(v, 0,0),(0, \mathrm{a}, 0)$, and $(0,0, d)$ can be used to estimate the three- dimensional variables separately. For the VAD point set $(v, 0,0)$ the Parzen-Borel kernel estimation is used, which is given by:

$$
f_{n}(x)=\frac{1}{n h_{n}} \sum_{i=1}^{n} K\left(\frac{x-v_{i}}{h_{n}}\right)
$$

Kernel estimation is a type of the nonparametric statistical methods to determine $K(u)$, which is a uniform density distribution function on $[-1,1]$. Thus, the kernel estimate in Eq.(22) is degenerated into an average, where $K(u)$ is usually used with the forms of $K=\frac{1}{2}$, Epanechikov kernel of $\frac{3}{4}\left(1-t^{2}\right), t \in[-1,1], \frac{5}{16}\left(1-t^{2}\right)^{2}, t \in[-1,1]$, and Gaussian of $\frac{1}{\sqrt{2 \pi}} e^{-\frac{1}{2} t^{2}}$. The best distribution of the function is Gaussian as shown in Fig. 7.

Through the definition of the kernel and the outer shell of the perceptual concept, the definition of the neighborhood-based method is used for the point set of different forms. At the same time, the outer layer of the emotional cell element is represented by the approximate set to express the characteristics of the soft film. Secondly, in the definition of the density function part, if the cell element of the VAD sentiment space has a single point set form, a single Gaussian density 
function is used. Also, for the planar form, the Gaussian mixture model is used. Through the iterative method, the parameters to be determined can be estimated to describe the distribution of the point set of the VAD emotion space [33].
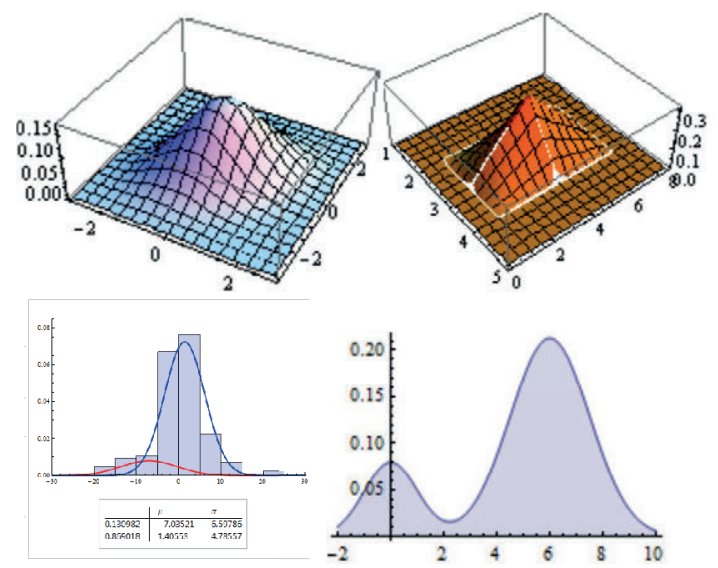

Fig. 7. An example of Gaussian distribution for the dimensional adjectives grouping model.

\section{Fuzzy Perceptual Inference Using the VAD Model for Clustering Evaluation}

\section{Fuzzy Evaluation Process}

When using fuzzy inference rules, the conditional statement "IF $\mathrm{x}$ is $\mathrm{A}$, THEN $\mathrm{y}$ is $\mathrm{B}$ " is converted into fuzzy relations rules. The Linguistic Variable (LV) was subsequently introduced for translating the natural language to a membership of fuzzy set as shown in Fig. 8.

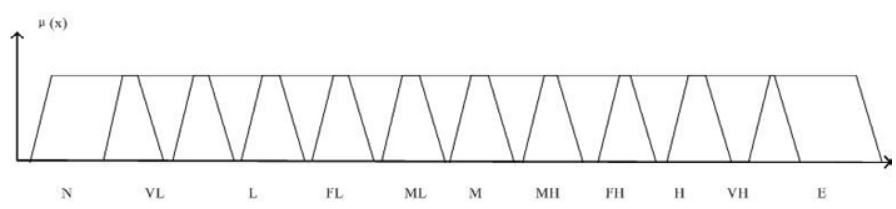

Fig. 8. Linguistic variables and their membership functions.

The fuzzy sets have been studied following fuzzy systems development rapidly. The selection of implication operators and fuzzy reasoning is closely related to the effect associated to the triangular norms. Besides, the implication operators study the fuzzy reasoning and fuzzy logic combined [34]-[35]. Therefore, the purpose is to accompany each other based on the triangular norms and implication operators to establish a new form of fuzzy propositional calculus system [36]. In the basic form of the production $P \rightarrow Q$ or "IF $P$ THEN $Q$ ", $P$ is a prerequisite for production(front piece), which gives the possibility to use a production prerequisite based on a logical combination to form; $Q$ is a conclusion or operation (post-production pieces). By using the Gaussian fusion as the density functions, the Fuzzy Inference System (FIS) rules fusion operation is of two "IF-THEN" rules integration. Supposed that,

RULE: IF " $\mathrm{x}$ is A", THEN " $\mathrm{y}$ is B", the assertion " $\mathrm{x}$ is A" satisfies the Gaussian distribution, which is given by:

$$
f(P, A, \sigma)=\frac{1}{\sqrt{2 \pi} \sigma_{A}} e^{-\frac{1}{2}\left[\frac{(P-A)^{2}}{\sigma_{A}{ }^{2}}\right]}
$$

Such as by Mamndini (Fig. 9), it is found that:

$$
\operatorname{Mamndini}_{F I S}=\operatorname{Min}\left(\frac{1}{\sqrt{2 \pi} \sigma_{A}} e^{-\frac{1}{2}\left[\frac{(P-A)^{2}}{\sigma_{A}^{2}}\right]}, \frac{1}{\sqrt{2 \pi} \sigma_{B}} e^{-\frac{1}{2}\left[\frac{(P-B)^{2}}{\sigma_{B}^{2}}\right]}\right)
$$

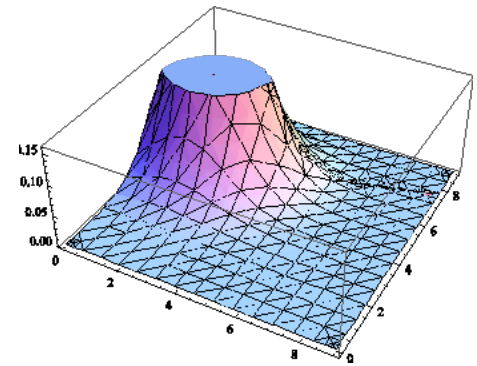

Fig. 9. IF-THEN rule by Mamndini Gaussian implication.

\section{FIS for Fusion and Decision Making}

Suppose that the $m$ knowledge rules in $v_{k}$ under Gaussian model (see formula (12)) conclude a particular assertion at the $\delta_{k}$ level, thus, we have that:

$$
\text { IF } x_{1} \text { is } v_{1} \text { and } x_{2} \text { is } v_{2} \text { and ..., and } x_{m} \text { is } v_{m} \text { THEN } I_{O} \text { is } V_{O} \text {, }
$$

where $\omega$ is the result, this can be simplified to the following rules:

$$
\begin{aligned}
& \text { IF } v_{1} \text { and } v_{2} \text { and, } \ldots \text {, and } v_{m} \text { THEN } \varpi\left(v_{1}, v_{2}, \ldots, v_{\mathrm{m}}\right) \\
& \text { IF } x_{1} \text { and } x_{2} \text { and, .., and } x_{m} \text { THEN } x_{1} \wedge x_{2} \wedge \ldots x_{m} \\
& \text { IF } d_{1} \text { and } d_{2} \text { and, } \ldots \text {, and } d_{m} \operatorname{THEN} d_{1} \wedge d_{2} \wedge \ldots d_{m} \\
& \text { IF } \rho_{1} \text { and } \rho_{2} \text { and, } \ldots \text {, and } \rho_{m} \operatorname{THEN} \varpi\left(\rho_{1}, \rho_{2}, \ldots, \rho_{\mathrm{m}}\right)
\end{aligned}
$$

Consider the density function $\delta_{k}$ in FIS and by letting $\Delta=\left[\delta_{1}, \delta_{2}, \ldots, \delta_{n}\right]$, it is found that:

\section{IF $\Delta$ THEN $\varpi(\Delta)$}

Since $\varpi(\Delta)$ is a Gaussian density function, the rule is re-labeled as "IF X THEN $f(X)$ ", and for this rule set, we have that:

$$
R_{i}: \text { IF X THEN } f_{i}(X)
$$

However, as $f(X)$ is a nonlinear function, it is difficult to find its minimum point under the Mamdani model, so we need to linearize $f(X)$ and use the nonlinear conjugate gradient algorithm to optimize the parameters of $f(X)$. To conduct sub-dataset indexing by some indices using fuzzy transformation, it is necessary to use the fuzzy factor analyst on a matrix type dataset [37].

\section{Proposed Model for Affective Clustering}

Basic hard c-means: First, the initial cluster centers are given, and all elements are assigned to each cluster according to the closest assignment principle to the cluster center. Afterward, to solve the new cluster center (element centroid) for each cluster, these steps are repeated until the centroid is no longer significantly changed, then the clustering is completed. The distance used depends on the nature of the data or project requirements; and the classification of distance can refer to the A-star algorithm overview and the Manhattan distance, diagonal distance and Euclidean distance can be considered. It is equivalent to solve the minimum problem of a global state function, which is the sum of the distances of each element to the nearest cluster center. The characteristics of this algorithm are, firstly, it does not necessary obtain a global optimal solution; while the initial cluster center does not meet the requirements, only locally optimal solution may be obtained [38]. For globally optimal solution, the algorithm changes the method name to k-means; secondly, the influence of noise points on clustering cannot be ruled out. Thirdly, the cluster shape is required to be nearly circular. The algorithm can be described as follows [39]: 


\author{
Algorithm 1: Hard c-means \\ Required: Number of clusters, epsilon [threshold] \\ Outputs: Clusters \\ a. Set cluster number's value k. \\ b. Choose the k cluster center randomly \\ c. WHILE center is not empty DO \\ Compute the mean or center of each cluster \\ Compute the distance of pixels and cluster's center \\ IF $\mid$ distance - center $\mid<$ epsilon THEN \\ Move points to the cluster. \\ ELSE: \\ Move points to the next cluster \\ ENDIF \\ Re-estimate the center \\ ENDWHILE \\ e. Output clusters
}

Extra fuzzy-c-mean: EFCM is a clustering algorithm based on a fuzzy number evaluation system that is also an improvement of the fuzzy c- mean (FCM) algorithm. The EFCM clustering model includes initialization, loop and output; firstly, the method is used to determine the initial clustering center to ensure the optimal solution, while the second step is to determine the degree of membership $U(i$, $j$ ) of each point to each cluster center. In which, $C^{(k)}$ is a weighted index. Thirdly, the system needs to determine the new cluster center and mark the change track of the cluster center to determine whether the changing amplitude of the cluster center is less than the given error limit. If not, it returns to the previous steps, otherwise it exits the loop. Finally, the system outputs the cluster center trajectory and clustering results. The characteristics of this algorithm are similar to ordinary k-means clustering. Full clustering is required, and noisy points cannot be distinguished. The center of clustering is more consistent, but the calculation efficiency is relatively low. The concepts of smoothing parameters and membership are adopted so that the points are not directly attached to a single cluster center. Algorithm 2 shows the process of the model [40]-[43].

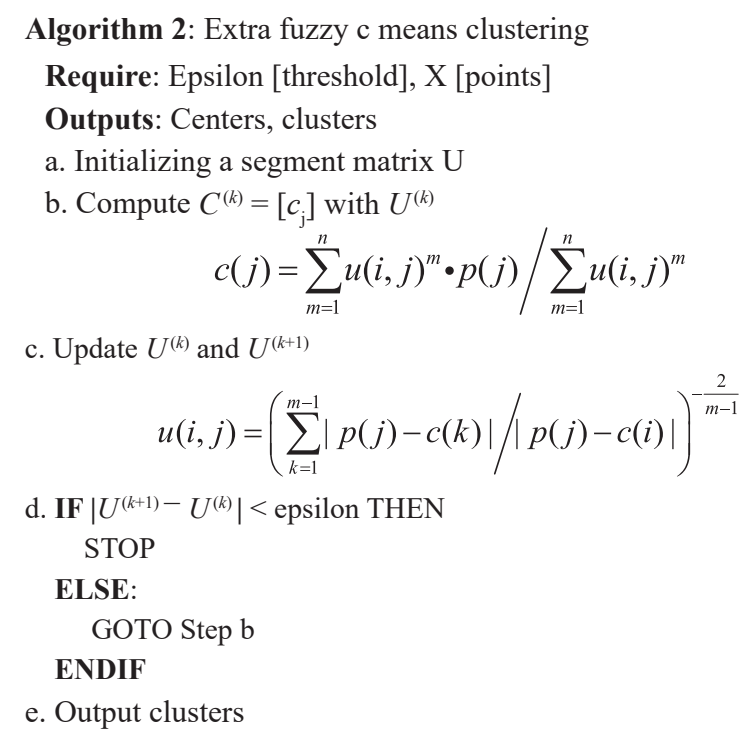

VAD-dimensional clustering: The Valence-Arousal-Dominance dimensional Clustering (VADdC) model was deployed based on hard c-mean and extra fuzzy c-mean. Each element in the initial state is a cluster, and the cluster with the smallest distance between clusters is merged each time until the number of clusters meets the requirements or merges more than $90 \%$. Similar to the Huffman tree algorithm, the union set also needs to be checked. For normal c-mean algorithms (HCM or EFCM), there are also several classifications of the distance definitions: including the minimum distance between cluster elements, the maximum distance between cluster elements, and the centroid distance of clusters. The characteristics of this algorithm are as follows.

a) The storage space consumed by the agglomeration clustering is higher than several other methods. The interference of the noise points can be eliminated, noise points may be divided into a cluster. Suitable for cases with irregular shapes and when complete clustering is not required.

b) The merging operation must have a merging limit ratio, otherwise excessive merging may cause all classification centers to aggregate, causing clustering failure.

Similar to the decision trees, the advantage of hierarchical clustering is that the entire tree can be obtained at one time, and the control of certain conditions, whether depth or width, is controllable. Several problems may occur, such as calculation on the division is determined and cannot be changed; and the cohesion/divisions combined is not "optimal" every time. However, the proposed VADdC algorithm is easy for local optimization and can be performed by appropriate random operations. It uses balanced iterative reducing and clustering. It first divides neighboring sample points into micro-clusters and then uses the c-means algorithm for these micro-clusters (Algorithm 3).

Algorithm 3: VADdC-valence arousal dominance dimensional clustering

Required: POINTS, points with index group, cluster numbers

Outputs: Groups, POINTS (clusters, centers)

a. POINTS $\leftarrow[\mathrm{v}, \mathrm{a}, \mathrm{d}]$ \#\# for each point in required VAD space

b. Number of Groups $\leftarrow$ index \#\# from 1 to index in range of length of POINTS

c. FOR index, point in POINTS:

IF type of point is required THEN

FOR index of the point in POINTS of group index ITERATE number of groups with point group

DELETE number of groups with points of index of point of the group

MERGE the groups with points and POINTS with index of point

ENDFOR

ELSE IF type of point is not required:

CALCULATE core point of group index with type of POINTS

COUNT the number of groups with core point group index

DELETE the number of groups with point group

The group of point $\leftarrow$ core point with group index

ENDIF

COUNT number of groups with sorted iterations using key $\leftarrow$ lambda

FOR key in several groups:

INCREASE $\mathrm{c} \quad \# \# \mathrm{c}=\mathrm{c}+1$

FOR point in POINTS:

IF group of the point is key*c THEN CONTINUE

\section{ENDIF}

\section{ENDFOR}

IF $\mathrm{c}>=$ cluster number THEN

BREAK \#\# exit the loop

ENDIF

ENDFOR

EDNFOR

d. Outputs POINTS, group \#\# clusters and centers 


\section{RESULTS AND DisCUSSION}

\section{A. Questionnaire System and Data Acquisition}

We developed a questionnaire system for data acquisition to be applied for evaluation. Chair styles are selected from an online shopping website, and the style has a list with values: glam, farmhouse, traditional, eclectic, bohemian, modern, global-inspired, coastal, American traditional, ornate glam, beachy, posh luxe, modern farmhouse, rustic, French country, industrial, ornate traditional, modern rustic, Scandinavian, sleek chic modern, mid-central modern, Asian-inspired, bold eclectic modern, cabin lodge, cottage Americana, nautical, tropical, and Victorian. The questionnaire system for the Mingstyle chairs for fuzzy perceptual evaluation was proposed by using (1-9) fuzzy numbers. An ancient furniture system in industrial design fields was evaluated. The relative dataset of results has been employed for clustering by using the VADdC model (shown in Table I).

TABLE I. Shape Classification and Their Sub-Shape Code In the Questionnaire System

\begin{tabular}{|l|l|l|l|l|}
\hline Shape Category & \multicolumn{4}{|l}{ Sub-shape } \\
\hline \multirow{2}{*}{ Top Rail (C1) } & C11 & C12 & C13 & C14 \\
\cline { 2 - 5 } & Scroll & Luoguo & Bow & Round \\
\hline \multirow{2}{*}{ Seat-Back (C2) } & C21 & C22 & C23 & C24 \\
\cline { 2 - 5 } & Screen & Comb-Teeth & Relief & Slatted \\
\hline \multirow{2}{*}{ Armrest (C3) } & C31 & C32 & C33 & C34 \\
\cline { 2 - 5 } & Square & Round & Openwork & Relief \\
\hline \multirow{2}{*}{ Foot (C4) } & C41 & C42 & C43 & C44 \\
\cline { 2 - 5 } & Carved Foot & Horseshoe & Square & Circle-Center \\
\hline
\end{tabular}

\section{B. Fuzzy Perceptual Evaluation using VADdC}

The adjective evaluation data is illustrated in Fig. 10 and 11, which show the result after grouping by using the VADdC algorithm. Fuzzy perceptual evaluation by using fuzzy number $[1,3,5,7,9]$ is applied on the shape of the Ming style chairs. Performance by using a fuzzy perceptual evaluation system is designed using 3 inputs, and 2 outputs basic Mamdani inference model based on the rule system as demonstrated in Fig. 12.

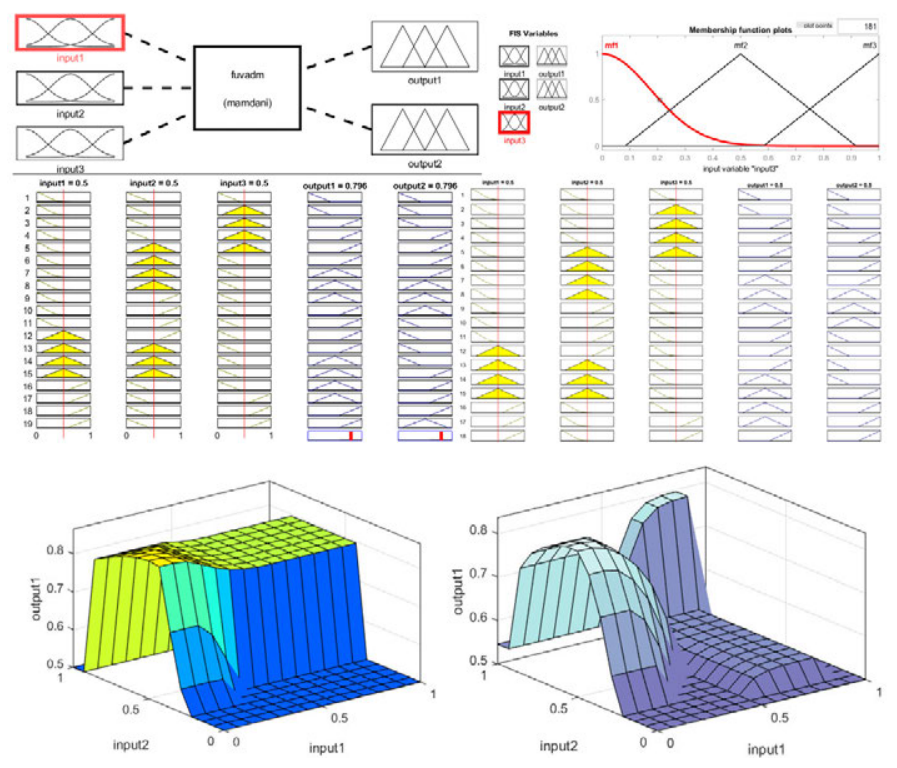

Fig. 12. 3-inputs 2-outputs basic fuzzy Mamdani inference model and rules.

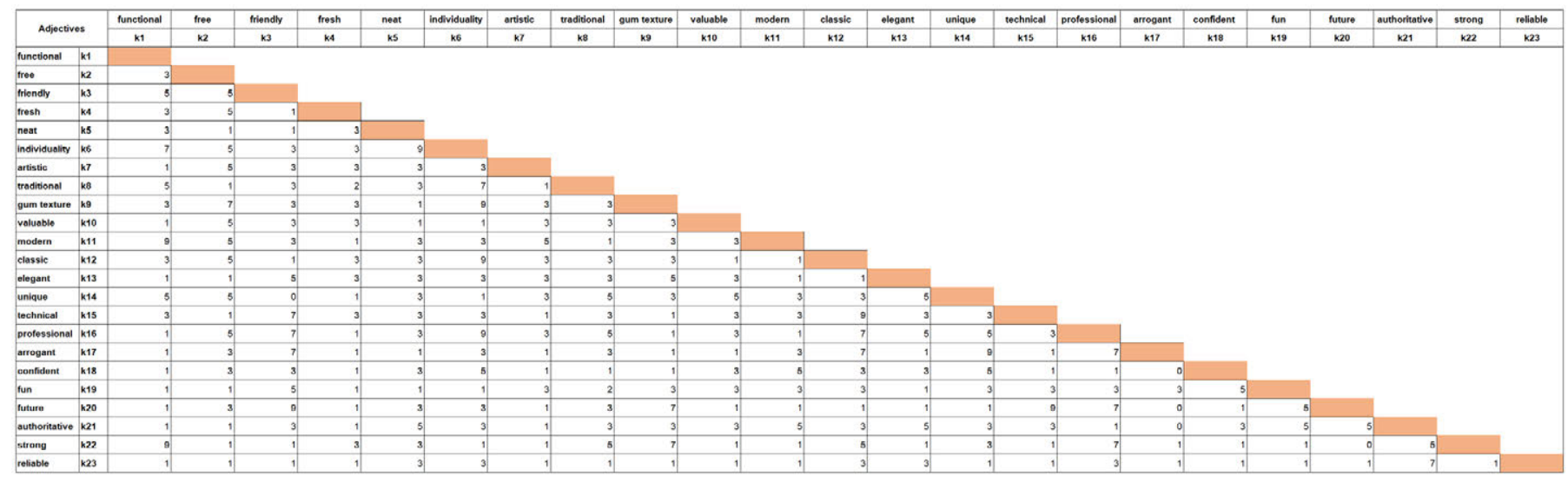

Fig. 10. Adjectives fuzzy perceptual evaluation before grouping.

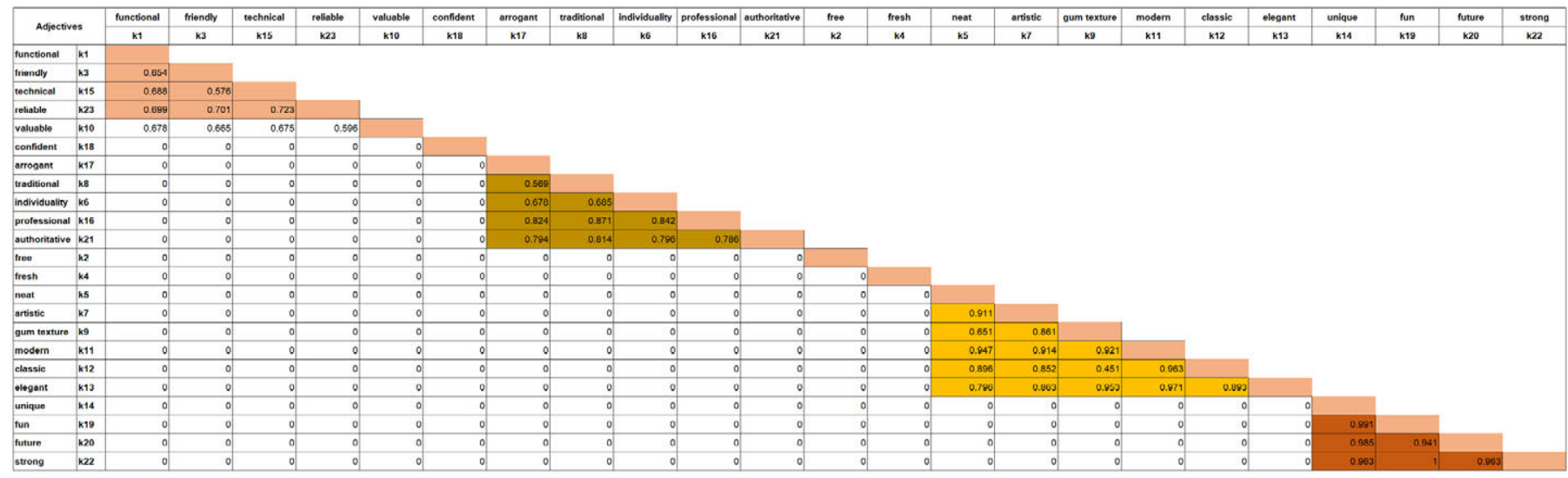

Fig. 11. Grouping adjectives by using clustering operators. 
TABLE II. Selected Adjectives Grouping and their Value Standard Deviation in VAD Dimensional Space

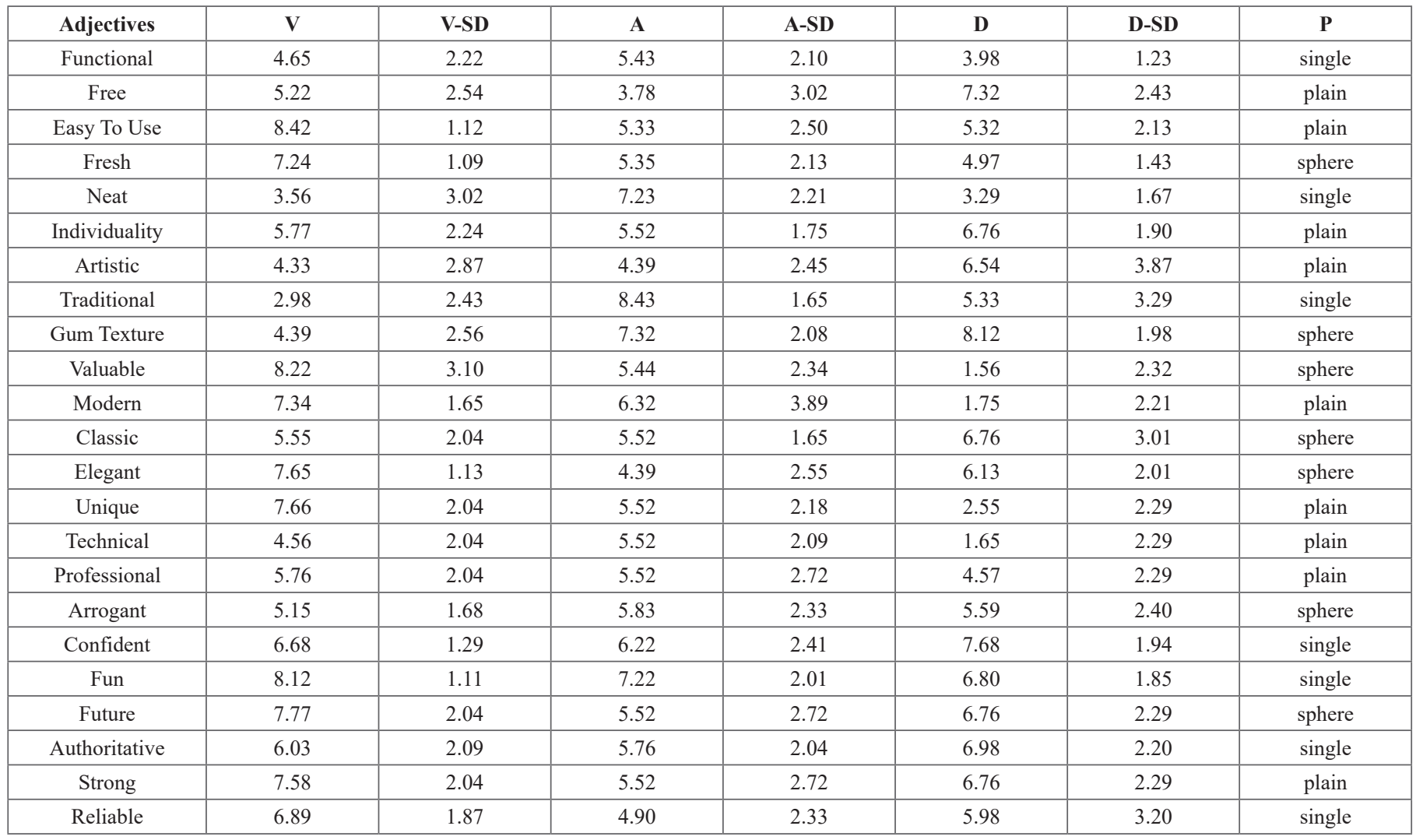

Table II shows the norms with VAD values from affective norm English Words (ANEW). Table III shows the comparing results of the VADdC model with FCM, K-mean, self-organization mapping network clustering (SOM), HCM, and EFCM. The VADdC performs $91.37 \%$ overall accuracy. Due to the superiority of the proposed method, different clustering and machine learning methods can be integrated with the proposed method to solve other applications as in [44]-[51].

TABLE III. COMPARING ANALYSIS OF HCM, EFCM AND VADDC

\begin{tabular}{|c|c|c|c|c|c|}
\hline $\begin{array}{c}\text { Clustering } \\
\text { Algorithms }\end{array}$ & $\begin{array}{c}\text { Fitness } \\
\text { value }\end{array}$ & $\begin{array}{c}\text { Inter- } \\
\text { cluster } \\
\text { Distance }\end{array}$ & $\begin{array}{c}\text { Intra- } \\
\text { cluster } \\
\text { Distance }\end{array}$ & $\begin{array}{c}\text { Elapse } \\
\text { time }\end{array}$ & $\begin{array}{c}\text { Accuracy } \\
\mathbf{( \% )}\end{array}$ \\
\hline FCM & 57.60 & 8.36 & 85.36 & 0.2865 & 79.68 \\
\hline K-mean & 47.23 & 7.56 & 76.37 & 0.3638 & 79.75 \\
\hline SOM & 59.62 & 10.36 & 122.35 & 0.4212 & 89.56 \\
\hline HCM & 60.89 & 8.566 & 89.32 & 0.3568 & 89.34 \\
\hline EFCM & 68.65 & 8.698 & 158.37 & 0.4251 & 90.07 \\
\hline VADdC & 17.53 & 9.210 & 126.36 & 0.4352 & 91.37 \\
\hline
\end{tabular}

\section{CONCLUSION}

In classical research, word vectors of features (terms, including words, words, phrases, etc.) are often used to build text vectors, and cluster analysis is performed based on the similarity between text vectors. To evaluate the clustering quality of the unsupervised clustering algorithm even in the case of the overlapping cluster centers, a VAD vector space model data was constructed for clustering analysis by studying the principle of clustering algorithms and applying clustering algorithms. For clustering a given set of objects, there can be multiple meaningful divisions due to the distance (or similarity) between objects, which has multiple implicit definitions. The present work constructed a VAD based model including distance, borders and type of centers. It also developed a rule-based inference system using fuzzy perceptual evaluation and introduced dimensional affective based VAD clustering called VADdC, taking successfully application on a dataset that has been acquired from an online questionnaire system.

The comparing analysis reported that the performance of the proposed method is better than others in the fitness of 17.53, elapse time of 0.4352 and $91.37 \%$ accuracy finally. In future work, highdimensional data can be involved, where the data distribution in a high-dimensional space may be very sparse, and highly skewed. In practical applications, it may be necessary to perform clustering under various conditions. Data grouping with clustering characteristics is very challenging. The most difficult question here is how to identify the "specific constraints" implicit in the problem we are trying to solve, and what algorithms should be used to best "fit" these constraints.

\section{ACKNOWLEDGMENT}

This work is supported by Science Foundation of Ministry of Education of China (Grant No:18YJC760099) and Zhejiang Provincial Key Laboratory of Integration of Healthy Smart Kitchen System (Grant No: 19080049-N)

\section{REFERENCES}

[1] Dey, N., Babo, R., Ashour, A. S., Bhatnagar, V., \& Bouhlel, M. S. (Eds.). "Social networks science: Design, implementation, security, and challenges: From social networks analysis to social networks intelligence", Springer. doi:10.1007/978-3-319-90059-9_1.

[2] Chiu, M.-C. and K.-Z. Lin. "Utilizing text mining and Kansei Engineering to support data-driven design automation at conceptual design stage", Advanced Engineering Informatics vol.38, pp.826-839, 2018, doi:10.1016/j.aei.2018.11.002.

[3] Ahmed, S. S., Dey, N., Ashour, A. S., et al. "Effect of fuzzy partitioning in Crohn's disease classification: a neuro-fuzzy-based approach", Medical \& 
Biological Engineering \& Computing, vol.55, no 1, pp.101-115, 2017, doi: 10.1007/s11517-016-1508-7

[4] Han, Z., et al. "Clustering and retrieval of mechanical CAD assembly models based on multi-source attributes information", Robotics and Computer-Integrated Manufacturing, vol.58, pp.220-229, 2019, doi:10.1016/j.rcim.2019.01.003

[5] Lacko, D., et al. "Product sizing with 3D anthropometry and k-medoids clustering", Computer-Aided Design, vol. 91, pp. 60-74, 2017, doi: 10.1016/j.cad.2017.06.004

[6] Li, W., et al. "A Comprehensive Approach for the Clustering of SimilarPerformance Cells for the Design of a Lithium-Ion Battery Module for Electric Vehicles", Engineering vol. 5, no. 4, pp. 795-802, 2019, doi: 10.1016/j.eng.2019.07.005

[7] Franco, M. A. "A system dynamics approach to product design and business model strategies for the circular economy", Journal of Cleaner Production, vol. 241, 118327, 2019, doi:10.1016/j.jclepro.2019.118327

[8] Chick, C. F. "Cooperative versus competitive influences of emotion and cognition on decision making: A primer for psychiatry research", Psychiatry Research, vol. 273, pp. 493-500, 2019, doi: 10.1016/j. psychres.2019.01.048.

[9] Mhetre, N. A., Deshpande, A. V., \& Mahalle, P. N. "Trust management model based on fuzzy approach for ubiquitous computing", International Journal of Ambient Computing and Intelligence, vol. 7, no.2, pp. 33-46, 2016, doi: 10.4018/978-1-5225-9866-4.ch022.

[10] Pleyers, G. "Shape congruence in product design: Impacts on automatically activated attitudes", Journal of Retailing and Consumer Services, 101935, 2019, doi: 10.1016/j.jretconser.2019.101935.

[11] Balakrishnan, J., et al. "Product recommendation algorithms in the age of omnichannel retailing -An intuitive clustering approach", Computers \& Industrial Engineering, vol. 115, pp. 459-470, 2017, doi: 10.1016/j. cie.2017.12.005.

[12] Büyüközkan, G. and M. Güler. "Smart watch evaluation with integrated hesitant fuzzy linguistic SAW-ARAS technique", Measurement vol. 153, 107353, 2019, doi: 10.1016/j.measurement.2019.107353.

[13] Lipor, J. and L. Balzano, "Clustering quality metrics for subspace clustering", Pattern Recognition, 107328, 2020, doi: 10.1016/j. patcog.2020.107328.

[14] Pandey, M. M. "Evaluating the strategic design parameters of airports in Thailand to meet service expectations of Low-Cost Airlines using the Fuzzy-based QFD method", Journal of Air Transport Management, vol. 82, 101738, 2019, doi: 10.1016/j.jairtraman.2019.101738.

[15] Qiao, W., et al. "A methodology to evaluate human factors contributed to maritime accident by mapping fuzzy FT into ANN based on HFACS", Ocean Engineering, vol. 197, 106892, 2019, doi: 10.1016/j. oceaneng.2019.106892.

[16] Hsu, C.-C., et al. "Relationship between eye fixation patterns and Kansei evaluation of 3D chair forms", Displays, vol. 50, pp. 21-34, 2017 doi: 10.1016/j.displa.2017.09.002.

[17] Cambria, E., Poria, S., Hussain, A., \& Liu, B. “Computational Intelligence for Affective Computing and Sentiment Analysis", IEEE Computational Intelligence Magazine, vol. 14, no. 2, pp. 16-17, 2019, doi: 10.1109/ MCI.2019.2901082.

[18] Gonzalez CB, García-Nieto J, Navas-Delgado I, Aldana-Montes JF, “A Fine Grain Sentiment Analysis with Semantics in Tweets", International Journal of Interactive Multimedia and Artificial Intelligence, vol. 3, no.6 pp. 22-28, 2016, doi: 10.9781/ijimai.2016.363.

[19] Kossaifi, J., et al. "AFEW-VA database for valence and arousal estimation in-the-wild”, Image and Vision Computing, vol. 65, pp.23-36, 2017, doi: 10.1016/j.imavis.2017.02.001.

[20] Jaeger, S. R., et al. "Valence, arousal and sentiment meanings of 33 facial emoji: Insights for the use of emoji in consumer research", Food Research International, vol. 119, pp. 895-907, 2018, doi: 10.1016/j. foodres.2018.10.074.

[21] Nalepa, G. J., et al. "Affective computing in ambient intelligence systems", Future Generation Computer Systems, vol. 92, pp. 454-457, 2018, doi: 10.1016/j.future.2018.11.016.

[22] Trabelsi, I., Bouhlel, M. S., \& Dey, N. "Discrete and continuous emotion recognition using sequence kernels", International Journal of Intelligent Engineering Informatics, vol. 5, no. 3 pp.194-205, 2017, doi:10.1504/ IJIEI.2017.086608.
[23] Sarkar, D., Debnath, S., Kole, D. K., \& Jana, P. "Influential Nodes Identification Based on Activity Behaviors and Network Structure with Personality Analysis in Egocentric Online Social Networks", International Journal of Ambient Computing and Intelligence, vol.10 no.4, pp.1-24, 2019, doi:10.4018/IJACI.2019100101.

[24] Pass-Lanneau, A., et al. "Perfect graphs with polynomially computable kernels", Discrete Applied Mathematics, vol. 272, pp. 69-74, 2018, doi: 10.1016/j.dam.2018.09.027.

[25] Person, O., et al. "Should new products look similar or different? The influence of the market environment on strategic product styling", Design Studies, vol. 29, no. 1, pp. 30-48, 2007, doi: 10.1016/j.destud.2007.06.005

[26] He, T., et al. "Curvature manipulation of the spectrum of Valence-Arousalrelated fMRI dataset using Gaussian-shaped Fast Fourier Transform and its application to fuzzy KANSEI adjectives modeling", Neurocomputing, vol. 174, pp. 1049-1059. 2015, doi: 10.1016/j.neucom.2015.10.025.

[27] Lee, W. and M. D. Norman. "Affective Computing as Complex Systems Science", Procedia Computer Science, vol. 95, pp. 18-23, 2016, doi: 10.1016/j.procs.2016.09.288.

[28] Salgado, S. and O. S. Kingo. "How is physiological arousal related to self-reported measures of emotional intensity and valence of events and their autobiographical memories?", Consciousness and Cognition, vol. 75, 102811, 2019, doi: 10.1016/j.concog.2019.102811.

[29] Scult, M. A. and A. R. Hariri. "A brief introduction to the neurogenetics of cognition-emotion interactions", Current Opinion in Behavioral Sciences, vol. 19, pp.50-54, 2017, doi: 10.1016/j.cobeha.2017.09.014.

[30] Wang, W. M., et al. "Multiple affective attribute classification of online customer product reviews: A heuristic deep learning method for supporting Kansei engineering", Engineering Applications of Artificial Intelligence, vol. 85, pp. 33-45, 2019, doi: 10.1016/j.engappai.2019.05.015.

[31] Zabotto, C. N., et al. "Automatic digital mood boards to connect users and designers with kansei engineering", International Journal of Industrial Ergonomics, vol. 74, 102829, 2019, doi: 10.1016/j.ergon.2019.102829.

[32] Hyun, K. H. and J.-H. Lee. "Balancing homogeneity and heterogeneity in design exploration by synthesizing novel design alternatives based on genetic algorithm and strategic styling decision", Advanced Engineering Informatics, vol. 38, pp. 113-128, 2018, doi: 10.1016/j.aei.2018.06.005.

[33] Kratzwald, B., et al. "Deep learning for affective computing: Text-based emotion recognition in decision support", Decision Support Systems, vol. 115, pp. 24-35, 2018, doi: 10.1016/j.dss.2018.09.002.

[34] Vučetić, M., et al. "Fuzzy functional dependencies and linguistic interpretations employed in knowledge discovery tasks from relational databases", Engineering Applications of Artificial Intelligence, vol. 88, 103395, 2019, doi: 10.1016/j.engappai.2019.103395.

[35] Bagherinia, A., et al. "Reliability-Based Fuzzy Clustering Ensemble", Fuzzy Sets and Systems, 2020, doi: 10.1016/j.fss.2020.03.008.

[36] Chen, Z., et al. "Explore and evaluate innovative value propositions for smart product service system: A novel graphics-based rough-fuzzy DEMATEL method", Journal of Cleaner Production, vol. 243, 118672, 2020, doi:10.1016/j.jclepro.2019.118672.

[37] Eustáquio, F. and T. Nogueira. "Evaluating the numerical instability in fuzzy clustering validation of high-dimensional data", Theoretical Computer Science, vol. 805, pp. 19-36, 2019, doi: 10.1016/j.tcs.2019.10.039.

[38] Hernández-Julio, Y. F., et al. "Fuzzy clustering and dynamic tables for knowledge discovery and decision-making: Analysis of the reproductive performance of the marine copepod Cyclopina sp", Aquaculture, 735183, 2020, doi: 10.1016/j.aquaculture.2020.735183.

[39] Li, W., et al. "Boosted K-nearest neighbor classifiers based on fuzzy granules", Knowledge-Based Systems 105606, 2020, doi: 10.1016/j. knosys.2020.105606

[40] Rani, P., et al. "A novel approach to extended fuzzy TOPSIS based on new divergence measures for renewable energy sources selection", Journal of Cleaner Production, vol. 257, 120352, 2020, doi: 10.1016/j. jclepro.2020.120352.

[41] García-Díaz, Vicente, Jordán Pascual Espada, Rubén González Crespo, B. Cristina Pelayo G-Bustelo, and Juan Manuel Cueva Lovelle. "An approach to improve the accuracy of probabilistic classifiers for decision support systems in sentiment analysis", Applied Soft Computing, vol. 67, pp. 822-833, 2018, doi: 10.1016/j.asoc.2017.05.038.

[42] Naik, Anima, Suresh Chandra Satapathy, Amira S. Ashour, and Nilanjan Dey. "Social group optimization for global optimization of multimodal functions and data clustering problems", Neural Computing and 
Applications, vol. 30, no. 1, pp. 271-287, 2018, doi: 10.1007/s00521-0162686-9.

[43] Hamzaoui, Y., Amnai, M., Choukri, A., \& Fakhri, Y. "Novel clustering method based on K-medoids and mobility metric", International Journal of Interactive Multimedia and Artificial Intelligence, vol.5, no.1, pp. 2933, 2018. doi: 10.9781/ijimai.2017.11.001.

[44] Cao, Luying, Nilanjan Dey, Amira S. Ashour, Simon Fong, R. Simon Sherratt, Lijun Wu, and Fuqian Shi. "Diabetic plantar pressure analysis using image fusion", Multimedia Tools and Applications, vol. 79, pp.11213-11236, 2020, doi: 10.1007/s11042-018-6269-x.

[45] Mondragon, Victor M., Vicente García-Díaz, Carlos Porcel, and Rubén González Crespo. "Adaptive contents for interactive TV guided by machine learning based on predictive sentiment analysis of data", Soft Computing, vol. 22, no. 8, pp. 2731-2752, 2018, doi: 10.1007/s00500017-2530-x.

[46] Tian, Zongmei, Nilanjan Dey, Amira S. Ashour, Pamela McCauley, and Fuqian Shi. "Morphological segmenting and neighborhood pixelbased locality preserving projection on brain fMRI dataset for semantic feature extraction: an affective computing study", Neural Computing and Applications, vol. 30, no. 12 pp. 3733-3748, 2018, doi: 10.1007/s00521017-2955-2.

[47] Li, Zairan, Kai Shi, Nilanjan Dey, Amira S. Ashour, Dan Wang, Valentina E. Balas, Pamela McCauley, and Fuqian Shi. "Rule-based back propagation neural networks for various precision rough set presented KANSEI knowledge prediction: a case study on shoe product form features extraction", Neural Computing and Applications, vol. 28, no. 3, pp. 613-630, 2017, doi:10.1007/s00521-016-2707-8.

[48] Pourvali, Mohsen, Salvatore Orlando, and Hosna Omidvarborna. "Topic models and fusion methods: a union to improve text clustering and cluster labeling", International Journal of Interactive Multimedia and Artificial Intelligence, vol. 5, no. 4, pp. 28-34, 2019, doi:10.9781/ijimai.2018.12.007.

[49] Shinde, Gitanjali Rahul, and Henning Olesen. "Beacon-Based Cluster Framework for Internet of People, Things, and Services (IoPTS)", International Journal of Ambient Computing and Intelligence, vol. 9, no. 4, pp. 15-33, 2018, doi:10.4018/JJACI.2018100102.

[50] Ali, Md Nawab Yousuf, Md Golam Sarowar, Md Lizur Rahman, Jyotismita Chaki, Nilanjan Dey, and João Manuel RS Tavares. "Adam deep learning with SOM for human sentiment classification." International Journal of Ambient Computing and Intelligence, vol.10, no. 3, pp. 92-116, 2019, doi:10.4018/IJACI.2019070106.

[51] Sengupta, Diganta. "Taxonomy on Ambient Computing: A Research Methodology Perspective", International Journal of Ambient Computing and Intelligence, vol.11, no. 1, pp.1-33, 2020, doi:10.4018/ IJACI.2020010101.

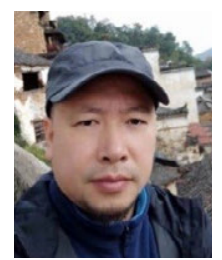

Wenlin Huang

Wenlin Huang, was born in Wenzhou City, Zhejiang Province, China in 1975. In 2000, he received a bachelor's degree in fine arts education from Wenzhou Normal University and a master's degree in fine arts from Tianjin Academy of Fine Arts in 2007. In 2010, he was employed as a lecturer in the Art and Design Department of City College of Wenzhou University, and in 2019, he was employed as an Associate Professor in the Arts and Crafts Department of Wenzhou Business College. His research direction is esthetic cognition and cultural design.

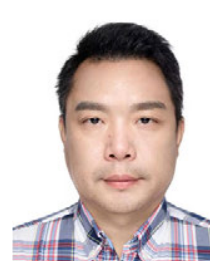

Qun Wu

Qun Wu, is an Associate Professor of Human Factor at the Institute of Universal Design, Zhejiang Sci-Tech University, China. He received his Ph.D. in College of Computer Science and Technology from Zhejiang University, China, in 2008. He holds a B.E. degree in Industrial Design from Nanchang University, China, in 2001, and a M.E. degree in Mechanical Engineering from Shaanxi University of Science and Technology, China, in 2004. His research interests include machine learning, human factor and product innovation design.

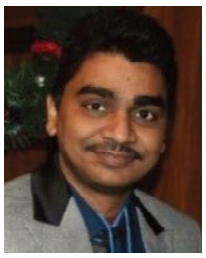

Nilanjan Dey

Nilanjan Dey, was born in Kolkata, India, in 1984. He received his B.Tech. degree in Information Technology from West Bengal University of Technology in 2005, M. Tech. in Information Technology in 2011 from the same University and Ph.D. in digital image processing in 2015 from Jadavpur University, India. In 2011, he was appointed as an Asst. Professor in the Department of Information Technology at JIS College of Engineering, Kalyani, India followed by Bengal College of Engineering College, Durgapur, India in 2014. He is now employed as an Asst. Professor in Department of Information Technology, Techno India College of Technology, India. His research topic is signal processing, machine learning, and information security. Dr. Dey is an Associate Editor of IEEE Access and is currently the Editor-in-Chief of the International Journal of Ambient Computing and Intelligence, and Series co-editor of Springer Tracts of Nature-Inspired Computing (STNIC).

\section{Amira S. Ashour}

He received her B.S. degree in Electrical Engineering (Electronics and Electrical Communications Engineering 'EEC') from Faculty of Engineering, Tanta University, Egypt in 1997, M.Sc. in Image Processing for Nondestructive Evaluation Performance, EEC Department, Faculty of Engineering, Egypt in 2000, and Ph.D. in Smart Antenna (Direction of arrival estimation using local polynomial approximation) from Faculty of Engineering, Tanta University, Egypt in 2005. Dr. Ashour is currently an Assistant Professor and Head of EEC Department, Faculty of Engineering, Tanta University, Egypt, since 2016. She is a member in the Research and Development Unit, Faculty of Engineering, Tanta University, Egypt. She is the ICT manager of Huawei-Tanta Academy, Tanta University. She was the Vice-chair of Computer Engineering Department, Computers and Information Technology College (CIT), Taif University, KSA for one year from 2015. She was the Vicechair of Computer Science Department, CIT College, Taif University, KSA for 5 years till 2015. Her research interests include image processing and analysis, medical imaging, computer-aided diagnosis, signal/image/video processing, machine learning, smart antenna, direction of arrival estimation, targets tracking, inverse problems, optimization, and neutrosophic theory. She is series Co-Editor of Advances in Ubiquitous Sensing Applications for Healthcare series, Elsevier.

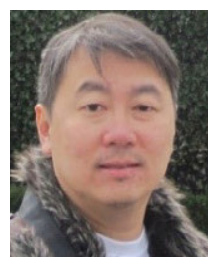

Simon James Fong

Simon Fong graduated from La Trobe University, Australia, with a 1st Class Honours BEng. Computer Systems degree and a PhD. Computer Science degree in 1993 and 1998 respectively. Simon is now working as an Associate Professor at the Computer and Information Science Department of the University of Macau, as an Adjunct Professor at Faculty of Informatics, Durban University of Technology, South Africa. He is a co-founder of the Data Analytics and Collaborative Computing Research Group in the Faculty of Science and Technology. Prior to his academic career, Simon took up various managerial and technical posts, such as systems engineer, IT consultant and e-commerce director in Australia and Asia. Dr. Fong has published over 450 international conference and peer-reviewed journal papers, mostly in the areas of data mining, data stream mining, big data analytics, meta-heuristics optimization algorithms, and their applications. He serves on the editorial boards of the Journal of Network and Computer Applications of Elsevier, IEEE IT Professional Magazine, and various special issues of SCIE-indexed journals. Simon is also an active researcher with leading positions such as Vice-chair of IEEE Computational Intelligence Society (CIS) Task Force on "Business Intelligence \& Knowledge Management", TC Chair of IEEE ComSoc e-Health SIG and Vice-director of International Consortium for Optimization and Modelling in Science and Industry (iCOMSI).

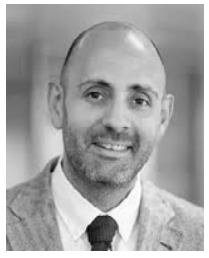

Rubén González Crespo

Dr. Rubén González Crespo has a PhD in Computer Science Engineering. Currently he is Vice Chancellor of Academic Affairs and Faculty from UNIR and Global Director of Engineering Schools from PROEDUCA Group. $\mathrm{He}$ is advisory board member for the Ministry of Education at Colombia and evaluator from the National Agency for Quality Evaluation and Accreditation of Spain (ANECA). $\mathrm{He}$ is member from different committees at ISO Organization. Finally, He has published more than 200 papers in indexed journals and congresses. 